\title{
From the Private into the Public: Privacy-Respecting Mobile Interaction Techniques for Sharing Data on Surfaces
}

\author{
Julian Seifert • David Dobbelstein • Dominik Schmidt \\ Paul Holleis . Enrico Rukzio
}

\begin{abstract}
Interactive horizontal surfaces provide large semi-public or public displays for co-located collaboration. In many cases users want to show, discuss, and copy personal information or media, which are typically stored on their mobile phones, on such a surface. This paper presents three novel direct interaction techniques (Select\&Place2Share, Select\&Touch2Share, and Shield\&Share) that allow users to select in private which information they want to share on the surface. All techniques are based on physical contact between mobile phone and surface. Users touch the surface with their phone or place it on the surface to determine the location for information or media to be shared. We compared these three techniques with the most frequently reported approach that immediately shows all media files on the table after placing the phone on a shared surface. The results of our user study show that such privacy preserving techniques are considered as crucial in this context and highlight in particular the advantages of Select\&Place2Share and Select\&Touch2Share in terms of user preferences, task load, and task completion time.
\end{abstract}

Keywords Interactive surfaces - mobile phones · interaction technique $\cdot$ data sharing.

\footnotetext{
J. Seifert · D. Dobbelstein · E. Rukzio

Ulm University, Ulm, Germany

E-mail: $\{$ first $\} .\{$ lastname $\} @$ uni-ulm.de

D. Schmidt

Hasso-Plattner-Institut, Potsdam, Germany

E-mail: dominik.schmidt@hpi.uni-potsdam.de

P. Holleis

DOCOMO Euro Labs, Munich, Germany

E-mail: holleis@docomolab-euro.com
}

\section{Introduction}

Interactive horizontal surfaces enjoy increasing popularity for all kinds of usages such as sharing and viewing of media, planning trips, browsing, or gaming. The constant increase in terms of technical features and the decrease of the price for such surfaces will lead to their pervasive usage for example at home, in offices, in hotels, in lounges, or in public buildings such as schools, universities, or libraries within the next decade. Their large size and multi-touch capabilities support in particular co-located collaborative interactions (e.g., [8, 21]). However, this also raises various privacy related questions when considering the information that could be displayed or stored on them. In contrast to mobile phones, interactive surfaces are public or semi-public devices and anyone nearby can see what is displayed.

The use of interactive surfaces for displaying, discussing and sharing private media (e.g., pictures) or information stored on the user's mobile phone (e.g., contacts, address information, or documents) is a frequently discussed scenario $[12,13,19,23]$. Here, a mobile phone needs to first establish a connection to the interactive surface and then, for instance, all pictures stored on the device $[12,23]$ or a thumbnail view of the pictures [13] can directly be shown at the table. Another possibility is that the user remotely selects information in private on the mobile phone before it is shown on the surface [2].

It is likely that most users store information on their mobile devices that they do not wish to show or share with others. This depends on the location in which the interactive surface is placed, the current situation, the relationship to the bystanders and the information to be shared. This might range from settings at home where one wants to share holiday pictures with close family 


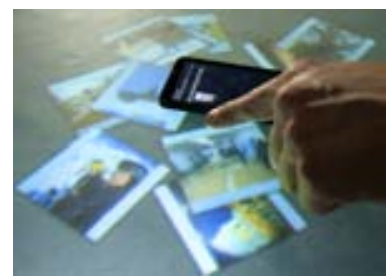

(a)

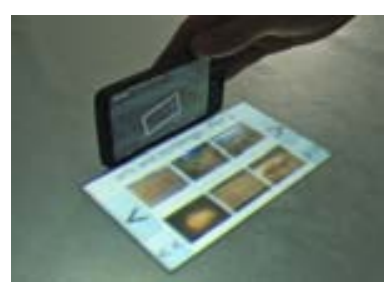

(c)

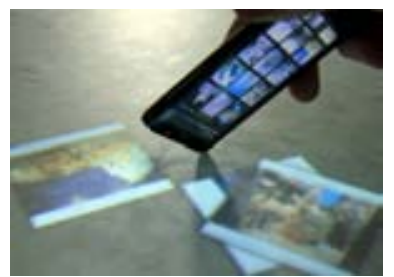

(b)

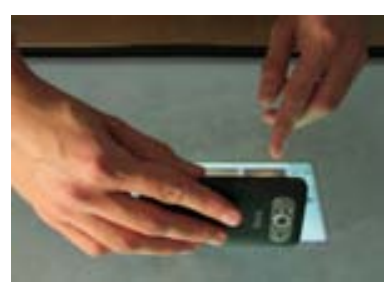

(d)
Fig. 1 Interaction techniques for privacy preserving sharing of data on interactive surfaces. a) Select\&Place2Share. b) Select\&Touch2Share. c) and d) Shield\&Share.

members to public settings in a hotel lobby where one wants to share only pictures of recently visited sights. Therefore, as users decide depending on the current context which data are appropriate for sharing with the current audience, effective means are required for selecting which data is to be shared. In particular, smart phones and their camera feature allow users to create large numbers of photos in diverse contexts. Interaction techniques are required that allow users to select from a large number of photos what they wish to share within a specific context. To address this, we designed and developed Select\&Place2Share and Select\&Touch2Share. Both techniques enable pre-selection of information on the mobile phone before showing it on the table (initiated by touching or placing the phone on the table as shown in Figure 1(a) and 1(b)). In the third technique, Shield\&Share, the user touches the surface with the side-edge of the phone so that the phone is placed like a viewing shield (see Figure 1(c) and 1(c)). On the phone's screen, the user can see a high-resolution preview of the selected file. At the same time, on the area right in front of the mobile phone facing the user, thumbnail views with navigation controls are displayed. For sharing a photo, the user simply drags the corresponding thumbnail from the menu bar at the bottom of the phone onto the public surface area. There, the photo is displayed visible for everyone around the surface.

The main contributions of this work are the three novel interaction techniques Select\&Touch2Share, Select\&Place2Share, and Shield\&Share that were influenced by previous work in this area, and the results gained from a comparative user study. The results indicate that users highly appreciate interaction techniques that support protecting their privacy. Further, users prefer interaction techniques that allow them to specify which items to share while the phone is held in the their hand.

\section{Related Work}

The research related to our work can be classified into four categories: (1) integration of personal devices (e.g., mobile phones) and shared displays (e.g., interactive surfaces and public displays), (2) extending and augmenting displays through connecting multiple devices, (3) privacy issues that arise from using personal devices in collaborative settings, and (4) direct touch interactions of mobile phones on interactive surfaces.

Integrating personal and shared devices. Allowing users to interact in a seamless way with their personal mobile devices and large shared displays has been studied in a wide range of works [1]. The main advantage is that accessing and displaying data (that are stored on the personal device) on large displays facilitates co-located collaboration of multiple users. Interaction with large displays could be direct (e.g., touching the display with the mobile phone) or indirect (e.g., using the mobile phone as a remote control). An example for indirect interactions is the work by Greenberg et al. who addressed the challenge how users can easily switch between individual work on their personal devices and share data on a public display [4]. Another example that falls in this category is the Pebbles system which supports interaction across several devices such as personal digital assistants (PDA) and public displays [14]. Chehimi and Rukzio presented an approach for sharing personal data on interactive surfaces where data is transmitted from the personal mobile phone to user proxies on the interactive surface [2].

One of the first examples for direct interactions in this context is hyperdragging, which allows users transmitting data from one device to another [16]. Using BlueTable, users connect their mobile phones and a shared interactive surface by placing them on the surface [23]. Furthermore it was demonstrated how data such as photos stored on the phone can be presented on the surface: once the phone is placed on the surface, the photos get 'spilled' out on the surface. A similar approach is presented by the Microsoft Mobile Connect Sample Application [13]: once a mobile phone is connected to the surface, the photos stored on the phone become accessible on the surface through an album widget. It allows users to browse through the photos. How- 
ever, spilling out all photos from a mobile phone onto the surface or making them accessible through a photo browser on the surface can result in unintended revealing of private photos. In addition, the initial transfer of all photos can result in significant waiting times for the user.

Extending and augmenting displays. Hinkley et al. presented stitching, a technique for combining multiple displays [7]. This technique allows users to place their tablet computers next to each other on an interactive surface and perform gestures across the device boundaries. The concept of Shield\&Share is related to stitching as it also allows users to connect two independent devices resulting in an augmented display with a combined user interface. Shield\&Share makes a significant contribution as it addresses privacy aspects when combining personal and public devices. The concept of expanding user interfaces across multiple displays also found application in multi-screen mobile phones (e.g., [10]). Expanding the user interface dynamically is also possible through Shield\&Share as users use their mobile phone and connect it to an interactive surface.

Privacy and sharing personal data on public displays. Users interacting on shared surfaces face challenges regarding privacy issues. Wu and Balakrishnan introduce the usage of the non-dominant hand to shield information displayed on the table from others while using the dominant hand to perform interactions in the shielded area [24]. Kim et al. showed that shielding a small area on the surface from the view of other users supports entering private information such as personal identification numbers [9]. Another privacy relevant issue arises from combining personal mobile phones of users with shared displays as users store large amounts of data on their personal devices [20]. Therefore, users should be in control of what data are shared. Shoemaker and Inkpen addressed the challenge of displaying private information within the context of a shared display by making certain information only visible to users with the corresponding access rights [22]. This approach requires users to wear shutter glasses that are connected to the display which allows displaying an individual view to each user. In contrast, Shield\&Share does not require users to use additional hardware but their mobile phones. With Ubitable, Shen et al. presented a system that allowed users to share and exchange data on an interactive surface [21]. Users could decide on a personal device (a laptop computer) which data should be transferred to the surface. The data appeared first in the private area on the surface, which could only be accessed by the user itself. Thus, the user is in control of what information is disclosed at all times.
Direct touch interaction techniques. Shield\&Share, Select\&Touch2Share, and Select\&Place2Share are based on users touching an interactive surface with their mobile phones. This has the advantage that the user can specify through a direct touch where some data should be placed or what data should be selected. Through this it is possible to define which data should be copied from which device to which other device by means of only one interaction. This simple task requires many steps using other technologies like Bluetooth [19]. The concept of direct touch interactions of one device with a public display was first shown by Rekimoto who presented Pick-and-Drop, an interaction technique that allows the user to directly pick up objects from one device and drop them on another device by touching objects directly with a pen [15]. Schmidt et al. demonstrated with their PhoneTouch system that this approach can also be applied to mobile phones and interactive surfaces, allowing to transmit data from the mobile phone to the surface and vice versa [17].

\section{Data Sharing Concepts}

Two aspects are of particular relevance when designing privacy respecting interaction techniques for sharing data between mobile phones and interactive surfaces. First, the ability for users to select ad-hoc what data to share is crucial. In particular, it may not be sufficient to priori classify data as public versus private since the changing usage context determines what is considered sensitive and worth protecting. Second, it is important to consider the phone's location during the sharing process; it can remain in the user's hand or may be placed on the interactive surface. Which interaction techniques users prefer and how well they support users to protect their privacy are open questions and need to be investigated. Therefore, we evaluated these interaction techniques in a comparative study.

In the following, we first discuss a technique commonly found in the literature to serve as baseline for our comparison. We then introduce three new direct touch interaction techniques that enable novel ways of sharing data stored on mobile phones on interactive surfaces.

\subsubsection{Place2Share}

The baseline interaction technique, Place2Share, builds on the BlueTable concept [23] and has been adopted by others (e.g., [12]). Place2Share consists of only one step: users place their mobile phones on the interactive surface. As soon as this event is detected by the system, all data (e.g., images) stored on the mobile phone are transmitted to the surface. There, the data is displayed 


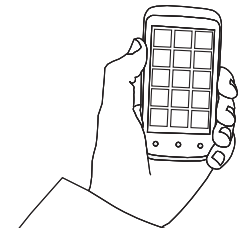

(a)

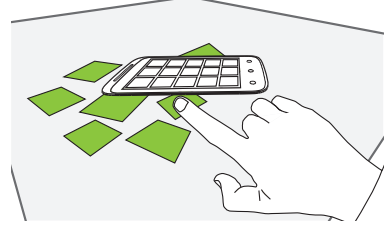

(b)
Fig. 2 Place2Share allows users to share all their data stored on the phone (a) by placing it on a surface. As the phone is placed, the data is transmitted to the surface and displayed around the phone (b).

around the mobile phone (see Figure 2(a)). Now the user can select items to interact with through touchbased interaction. In the opposite direction, users can transfer data from the surface to the phone by dragging a picture very close to the phone.

Place2Share does not allow for selecting data that are intended to be shared. Therefore, the interaction is very straightforward. Users, however, cannot protect the privacy of their data as the photos are instantly shown on the surface. Initially, the phone is held in the hand and then placed on the surface. This allows for touch-based interaction with two hands on the surface.

\subsubsection{SelectEPlace2Share}

Select\&Place2Share is a modified version of the Place2Share interaction technique. The user can make a selection of data items to be shared on the surface beforehand. The selection is made on the phone by marking items as public through touching them (see Figure $3(\mathrm{a})$ ). Touching marked items again changes the state back to private. When the user places the mobile phone on the surface the items that are contained in the public folder are transmitted to the surface and displayed around the phone (see Figure 3(b)). In the opposite direction, the user can transfer data from the surface to the phone by dragging a picture very close to the phone.

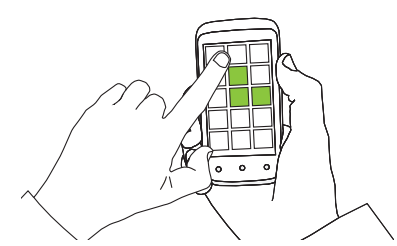

(a)

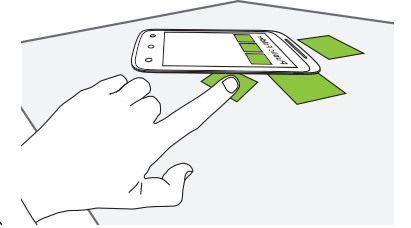

(b)
Fig. 3 Select\&Place2Share allows users to select items that are intended for sharing (a). Then users place their phone on the surface and selected items are displayed around the phone (b).
This interaction technique protects the users' privacy as they have to explicitly define what data is going to be shared. The selection is made while the phone remains in the hand of the user. Thus, others cannot observe what is selected and it is not possible to assess how much data is stored on the user's phone. The phone is then placed on the surface which allows two-handed interactions on the surface.

\subsubsection{SelectETouch2Share}

The interaction technique Select\&Touch2Share is based on direct touch interactions between the mobile phone and the interactive surface previously reported (e.g., $[15,17,19])$. In order to apply this interaction technique, the user first makes a selection of data on the mobile phone (see Figure 4(a)); then the user performs a touch with the phone on the interactive surface (see Figure $4(\mathrm{~b}))$. As this event occurs, the selected data is transferred to the surface and displayed around the touch location (see Figure 4). For transferring data back from the interactive surface to the phone, users touch the corresponding item on the surface with the phone.

Similar to Select\&Place2Share, the selection of data that are intended for sharing is done through marking items as public by touching them on the phone screen beforehand. As a result, the selection can be made in private without risking to disclose any private data. In contrast to the previous techniques, the phone remains in the hand of the user throughout the whole interaction process. As a consequence, users can interact only using one hand with the surface while the other one holding the phone is occupied. Yet, the phone remaining in the hand of the user additionally supports the protection of the user's data as the phone cannot be viewed or accessed by others.

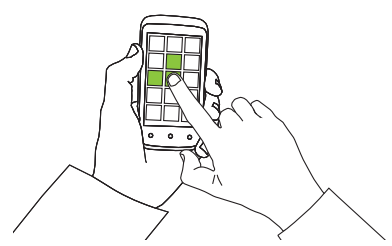

(a)

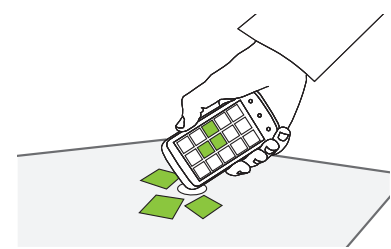

(b)
Fig. 4 Select\&Touch2Share allows users to make a selection of items that are intended for sharing (a). Then they touch the surface with their phone and selected items are displayed around the phone (a). 


\subsubsection{ShieldEShare}

The fourth interaction technique Shield\&Share was inspired by previous work $[24,18]$ and allows the user to share data on an interactive surface while the phone is placed like a viewing shield (see Figure 5). The concept of shielding private information with the non-dominant hand is well-known and used in many other areas such as typing in a code when interacting with an ATM. As the user places the phone on the surface, a menu bar appears at the bottom of the phone, containing small thumbnails representing data items. The phone itself prevents other users from seeing details of the thumbnails behind the phone. When the user touches a thumbnail in the menu bar displayed on the interactive surface, a detailed preview of the data is displayed on the phone's screen. In case of photos, a high-resolution preview is displayed. For sharing data with others, the user drags the corresponding thumbnail out of the menu bar onto the public surface area. For transferring data from the surface to the phone, the user drags items from the surface into the menu bar displayed on the interactive surface at the bottom of the mobile phone.

Browsing through the data and selecting an item takes place while the phone remains in the hand of the user, but is connected with the surface at the same time. The user's privacy is protected as only data items explicitly dragged onto the surface are shown to others. Of course, depending on the location of bystanders, the phone might shield only parts of the menu bar. Therefore the thumbnails need to be rendered in a low resolution in order to additionally prevent others from seeing details of private data.

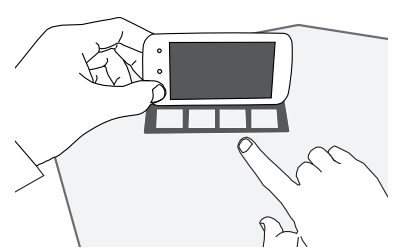

(a)

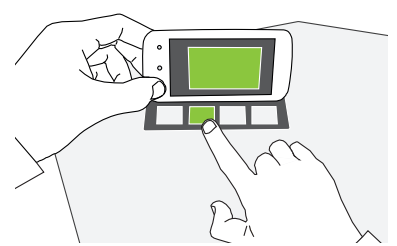

(c)

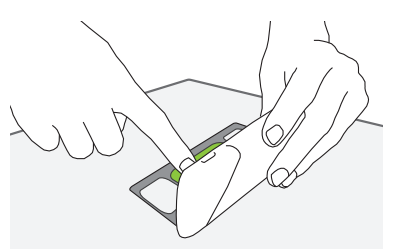

(b)

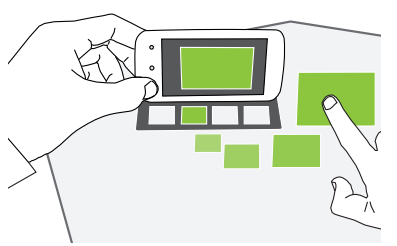

(d)
Fig. 5 Shield\&Share requires users to place their phone on the surface like a viewing shield ((a) and (b)). For sharing data, the user drags the item out of the bar onto the surface ((c) and (d)).

\section{System Implementation}

We implemented the discussed interaction techniques within a sharing application that allows users to view and exchange photos. As interactive surface, we used a system based on frustrated total internal reflection (FTIR) [5]. The interactive surface has a resolution of $1280 \times 800 \mathrm{px}$ and is operated through a computer running Windows 7 (64 bit). The graphical user interface on the surface is implemented using the Microsoft Surface 2.0 SDK. We used a HTC HD7 smartphone, running the Windows Phone 7 (WP7) operating system. We applied a client-server model, whereas phones and the surface applications were connected to a surfaceserver managing communication and data transfer (via TCP) between connected clients and detection of direct touch events. When the phone client is started, the connection to the surface server is automatically established and remains until the user exits the phone application. The hardware ID of the phone allows the system to distinguish between connected phones which enables multiple users to use the system simultaneously. For direct touch interaction between mobile phone and interactive surface, a time correlation-based touch detection was applied [17]. The mobile phone's microphone and accelerometer are used for detecting the bump event that occurs when touching the interactive surface. On the surface-side, visual blobs are detected. Both send detected events to the surface server for inspection. When the time difference between these events remains below a defined threshold, a successful phone touch was detected and the system knows where the surface has been touched by which mobile phone and corresponding images are transferred to this location. Images transferred from the mobile phone to the surface application remain there after the connection of the phone to the surface server is closed. Alternative options are removing them automatically after the connection is closed or allowing users to explicitly leave behind selected images.

Each interaction technique makes specific demands for the implementation of the photo sharing application. In the following, we illustrate the specific aspects for each of the implemented techniques.

\subsubsection{Implementation of Place2Share}

The first, baseline concept requires users to place their mobile phone on the surface (see Figure 6). Before doing so, the user touches the surface with the phone. As the phone touch is detected, a proxy appears on the surface that is associated with the mobile phone. Then, the user places the phone on this proxy. The phone's 


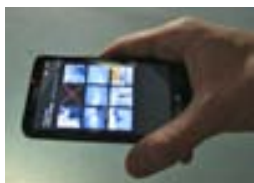

(a)

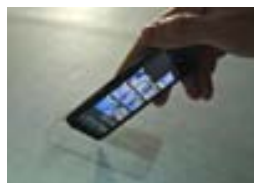

(b)

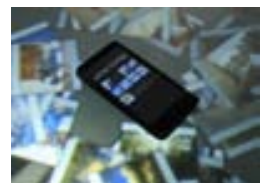

(c)
Fig. 6 Place2Share allows the user to place the phone on the surface. Once the phone is lying on the surface, all photos are copied from the phone to the surface.

accelerometer sensor is used for detecting that it has been placed on the surface (the values of the $\mathrm{z}$-axis have to reach a specific threshold and remain above this value for a defined period). Then all photos are sent to and displayed on the surface around the phone.

We selected this approach as base line as it appears in the literature and in demo applications for interactive surfaces (e.g., [11,23]). With Place2Share, users can transfer photos from the surface to their phones. This can be achieved by dragging photos on the surface close to the phone (see Figure 7). When the photo is downloaded, the phone displays the folder containing incoming photos. When photos are transferred to the phone, the folder Incoming Photos is displayed including the new photos.

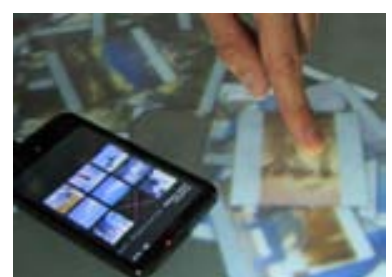

(a)

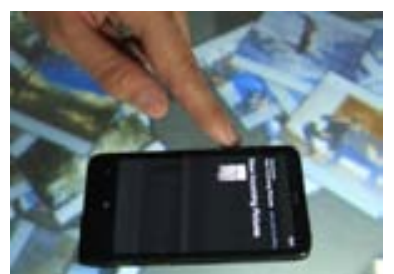

(b)
Fig. 7 Users can transmit data from the surface to their mobile by dragging items close to the phone (a). Added files are stored in an incoming folder (b).

\subsubsection{Implementation of SelectङPlace2Share}

The concept of Select\&Place2Share allows the user to select the photos to be shared before the phone is placed on the interactive surface. Therefore, the implementation offers an interface to mark photos as public (see Figure 8). References to these photos are displayed in the Public Folder. The user can deselect images that are not intended for sharing anymore. For placing the phone on the surface, the user performs a phone touch to create a proxy and places the phone on the latter. Transferring photos back from the surface to the phone works in the same way as with Place2Share illustrated previously.

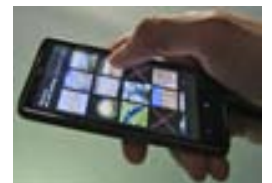

(a)

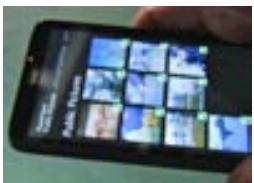

(b)

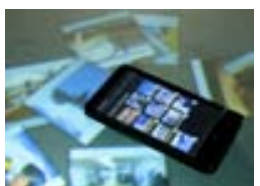

(c)
Fig. 8 Using Select\&Place2Share, the user first marks photos as public. These can be reviewed using the Public Folder. When the phone is placed on the surface, only public photos transferred to the surface.

\subsubsection{Implementation of SelectETouch2Share}

The implementation of Select\&Touch2Share also allows the user to specify which photos should be shared. Similar to Select\&Place2Share, users touch the tiles representing the photos they wish to share (see Figure 9). Once finished with the selection, they touch the surface with the phone to start transmitting the photos to the surface.

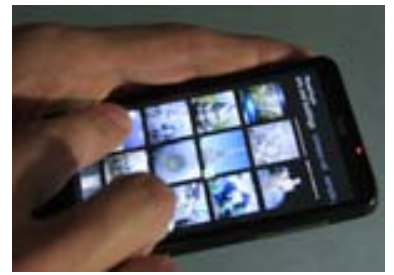

(a)

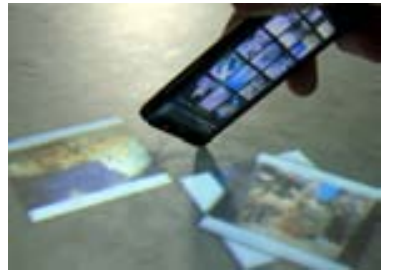

(b)
Fig. 9 Select\&Touch2Share allows users to first select a number of photos (a). When they touch the surface with their phone, the photos displayed around the touch location on the surface (b).

The user can place photos at a specific location on the surface as they are displayed around the location where the phone touched the surface. The user can upload photos from the surface to the phone by touching the desired photos displayed on the surface with the phone.

\subsubsection{Implementation of ShieldEShare}

In order to make use of Shield\&Share, the user first needs to pair the mobile phone with the interactive surface. To do so, the user touches the surface with one corner of the phone; then the user rotates the phone towards the surface until its side fully touches the surface (see Figure 10). This sequence of steps was chosen as the shape of the edge of the phone could not be detected in a reliable way by the surface. The main reason 


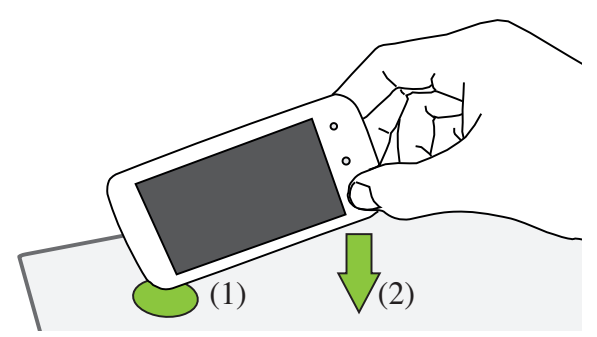

Fig. 10 To start the Shield\&Share interaction, first, the user has to touch the surface with the corner of the mobile phone. Then the phone is moved down on the surface so that the edge touches the surface.

is that buttons placed on the edge of the phone touch the surface in different ways depending on the angle of phone.

When the physical connection between phone and surface is successfully detected, the menu bar interface is displayed at the bottom of the phone on the surface. In our implementation, the orientation where the menu bar is displayed is determined based on the shortest distance to the edge of the surface screen. That is, the interface is displayed on the side of the phone that points towards the nearest surface border.

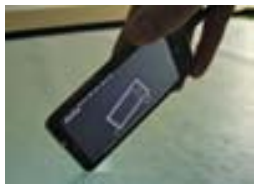

(a)

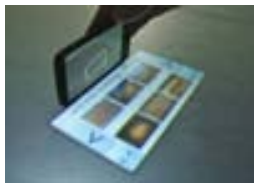

(b)

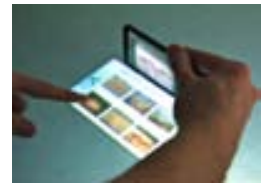

(c)
Fig. 11 Shield\&Share is initiated by touching the interactive surface with a corner of the phone. When the phone edge is touching the surface, the interface is displayed. Touching a thumbnail in the menu bar will start a preview on the phones display.

Figure 11 shows how to set up the menu bar interface of Shield\&Share. The menu bar displays two rows of photo thumbnails with a low resolution $(50 \times 50 \mathrm{px}$ per thumbnail). In addition, the menu bar contains two buttons on each side. One button is for switching to the next photo album, the other for selecting the next subset of photos from the current album. When the user touches a thumbnail, a high-resolution preview of the photo is displayed on the phone screen.

The size of the menu bar $(225 \times 133 \mathrm{px}$, which corresponds to $17.5 \times 10.3 \mathrm{~cm}$ ) was chosen to be large enough to contain at least six photo thumbnails. Due to the relatively low resolution of the interactive surface $(1280 \times 800 \mathrm{px})$ the thumbnails could not be smaller. Figure 12 shows the implementation of Shield\&Share
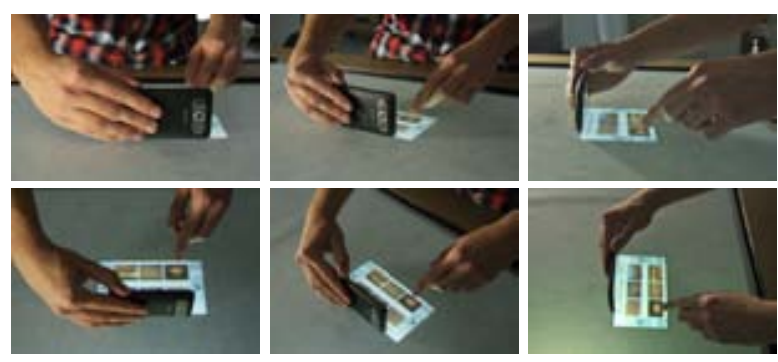

Fig. 12 Shield\&Share and how well it protects the user's privacy is depending on the angle and the height of view. This image series shows the use of Shield\&Share from two different heights and viewing angles.

from three different viewing angles: from a height of $160 \mathrm{~cm}$ and from a height of $190 \mathrm{~cm}$. It appears that only in one case the phone is capable of shielding the menu bar completely from the observers view. Users can share photos by dragging a thumbnail out of the menu bar onto the surface. Vice versa, photos from the surface can be added to the phone by dragging them over the menu bar and dropping them there (see Figure 13).

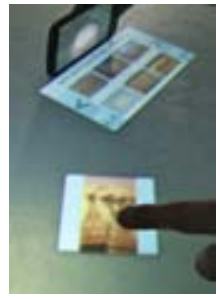

(a)

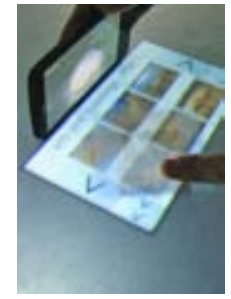

(b)

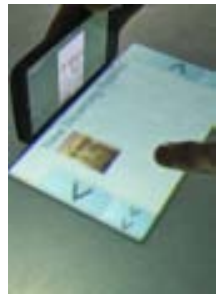

(c)
Fig. 13 Adding a photo from the surface to the mobile phone using the interaction technique Shield\&Share.

\section{Comparative Study}

We designed and conducted a user study in order to compare the three discussed privacy preserving interaction techniques (Select\&Place2Share, Select\&Touch2Share, and Shield\&Share) and to gain in-depth insights in how users experience them. The interaction technique Place2Share served as baseline as it does not support users to protect their privacy. In particular, the evaluation aims for providing insights about the effectiveness of support for privacy respecting data sharing, user acceptance, and usability aspects. We also looked at aspects such as perceived effort or task completion time to investigate potential effects of the new privacy preserving interaction techniques on the overall interaction task. We did not include a phone only option 
as a further comparative system as an interactive surface supports effective and efficient co-located collaboration and as phone based solutions suffer from the small screens designed for a single user.

We decided to evaluate the interaction techniques in the context of a photo sharing situation where the participants would share specific photos with another person. We selected this context as people understand easily in what ways photos can be regarded as private or sensitive.

\subsection{Study Procedure}

The participants took part individually. The study was organized in three phases. (1) The participants filled out a questionnaire regarding their general experience and usage of mobile phones and their photo sharing behavior. (2) They performed a series of practical tasks with all four interaction techniques preceded with a training phase. We used a within-subjects design so that each participant evaluated each interaction technique. The order in which the interaction techniques were selected was counterbalanced using Latin square. The order of the tasks was randomized. No time was required for transmitting the images between surface and phone in the study as they were already stored on those devices beforehand. After finishing all tasks with the respective technique, the users completed a questionnaire. (3) In the third phase, users ranked all tested interaction techniques with regards to interaction speed, privacy protection, and general preferences in a second questionnaire. We decided to use a photo sharing scenario for the user study in order to give the participants a well-known context for the practical tasks.

Participants were introduced to the practical tasks they were about to perform. During these, they had to search and show a number of photos that were stored on the provided mobile phone to the experimenter. In total, a set of 69 photos was prepared and stored on the mobile phone for the user study. These were organized in four photo albums (arts and buildings, winter holiday, camera roll, and the pre-installed sample photos). They also contained seven special images which the participants should not disclose to the investigator. The participants could recognize them easily as this were black images with a large red cross (see Figure 14). We considered asking the participants to provide own public and private photos for the study but this would have been very unrealistic as truly private pictures would not have been chosen by the participants and further, behavior of the participants would be influenced by different conditions. Considering that privacy is a very subtle notion depending on many factors such

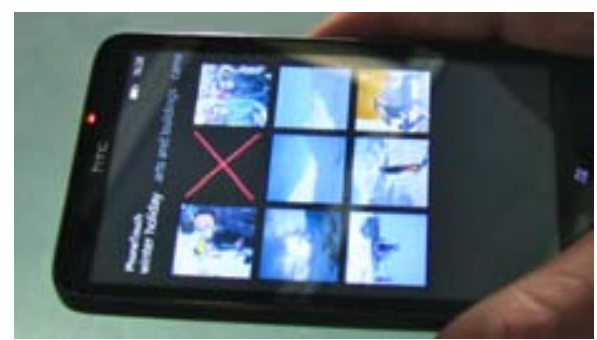

Fig. 14 Photos that were to be considered as private were represented by black images with a red cross.

as context and audience, this experimental condition can only simulate a sharing situation. However, it allows comparing the selected interaction techniques in terms of support to disclose a defined set of images.

In a training phase before the practical tasks, participants had time to familiarize themselves with the albums. Also, they were told to look up the photos that were to share in the upcoming tasks, to make sure that those interaction technique tested first would not strongly be affected.

In the following, participants were asked to perform the following sequence of tasks with each interaction technique. (1) "Please show me your photos of the Eiffel Tower and the Colosseum." (two photos). (2) "Could you please show me the photos you took of the train station that was water-flooded lately?" (four photos). (3) "Last winter we were skiing. Can you show me photos with me wearing this yellow helmet?" (two photos). (4) "Could you please add these photos to you phone, so that you can show them to our other friend?" (three photos).

A video camera mounted on the ceiling above the interactive surface recorded all sessions for capturing the interactions of the participants. Also, on the interactive surface, all events and interactions were logged.

We recruited 16 participants. Seven of them were female and their average age was 23 years $(21-27)$. The majority of the participants were students (11 undergraduates, 4 graduates). One participant was an employee. Six of the participants had a computer science background. The others had a background in humanities or economics.

\section{Study Results}

All participants used mobile phones with a photo camera for several years. Ten of them used a smartphone as their personal mobile phone. The participants reported to store a variety of different data on their phones such as music, messages (email, text), calendars, appointments, and photos. In particular, they stored in average 
174 photos $(S D=271)$ on their phones they brought with them to the study. These great differences in the number of stored photos are also reflected by the importance the participants attach to this feature of their mobile phones. On a five point Likert scale ( $5=$ very important), they rated the camera feature on average at $3.51(S D=1.40)$, while four rated it with 1 or 2 .

Participants assessed the frequency ( $5=$ very often) of showing photos they have on their mobile phones to other people with $3.20(S D=1.43)$. Similarly they rated the frequency how often they share photos with others $(M=2.51 ; S D=1.40)$. They reported to use Bluetooth, email, USB cable to PC, Facebook, and Dropbox channels for sharing photos with others, with Bluetooth sharing being named most often (6 times). Ten of the participants stated that they would hand their phone to other persons in order to show them certain photos. However, some added that they would hand their mobile phone only to friends. Four of the participants stated that they would not give their phone to other persons under any circumstances.

After each trial, participants rated the tested interaction technique using selected questions from the NASA TLX [6]; as questions before on a five point Likert scale ( $1=$ very low; $5=$ very high). Selected questions for comparison of interaction techniques were: Performance: How successful were you in accomplishing what you were asked to do? Effort: How hard did you have to work to accomplish your level of performance? Frustration: How insecure, discouraged, irritated, stressed, and annoyed were you? Physical demand: How physically demanding was the task? Mental demand: How mentally demanding was the task?

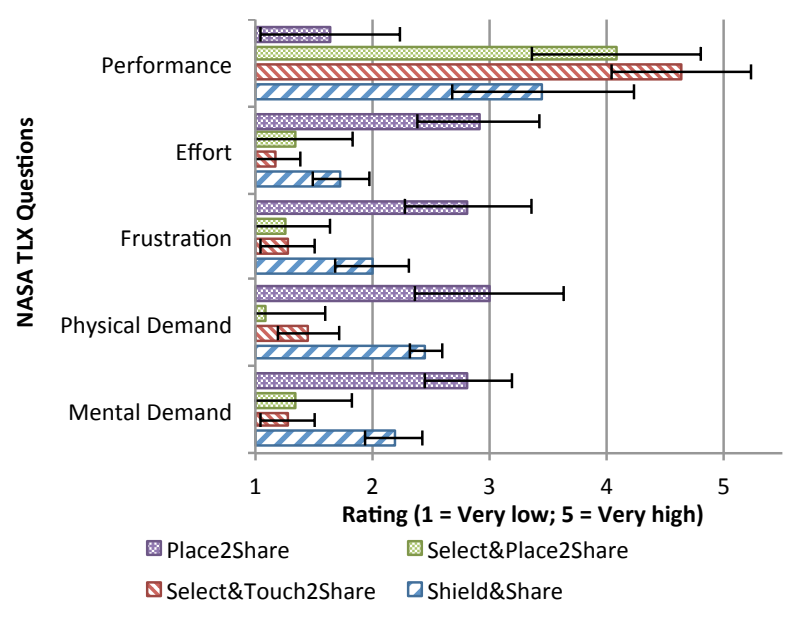

Fig. 15 Participants' estimations of the four evaluated interaction techniques based on the NASA TLX questions. Bars show the mean values; error bars indicate standard deviation.
Using Friendman's ANOVA we tested for differences between the techniques (level of significance $\alpha=0.05$ ) and used Wilcoxon's signed-rank test with Bonferoni correction for pairwise comparison where appropriate. Concerning the perceived level of performance the ratings were significantly different $\left(\chi^{2}(3)=20.81\right)$. Pairwise comparison showed that participants rated Select\&Touch2Share $(z=-1.9, p=0.003)$ and Select\&Place2Share $(z=-1.81, p=0.006)$ significantly higher compared to Place2Share. In regards of effort the ratings were found to be significantly different $\left(\chi^{2}(3)=\right.$ 15.62).Pairwise comparison showed that the perceived effort was higher with Place2Share compared to Select\&Place2Share $(z=1.54, p=0.03)$ and Select\&Touch2Share $(z=1.77, p=0.008)$. Concerning the perceived frustration level the ratings differ significantly $\left(\chi^{2}(3)=15.50\right)$.Pairwise comparison showed that the frustration level for Place2Share was rated significantly higher than for Select\&Place2Share $(z=1.72, p=$ $0.01)$ and Select\&Touch2Share $(z=1.45, p=0.03)$. Also, ratings concerning the physical demand differed significantly $\left(\chi^{2}(3)=16.35\right)$.It appears that the physical demand for using Select\&Place2Share was rated significantly lower than Shield\&Share $(z=1.45, p=0.04)$ and Place2Share $(z=1.9, p=0.003)$. Ratings for the perceived mental demand differ significantly $\left(\chi^{2}(3)=\right.$ 18.66).Pairwise comparison showed that Place2Share was rated to be significantly more mentally demanding as Select\&Touch2Share $(z=1.86, p=0.004)$ and Select\&Place2Share $(z=1.9, p=0.003)$.

These results show (see Figure 15) that Place2Share was consistently rated worst (e.g., least performance, highest effort etc). Main reason was a delay caused by the demand to render the 69 images after placing the phone on the surface. Furthermore, participants had to browse and search for the pictures which were spilled on the surface. Also, the phone was perceived as disturbing lying on the surface together with such a large number of photos.

Further, the results indicate that Shield\&Share required a higher effort, caused more frustration as well as a higher physical and mental demand compared to Select\&Place2Share and Select\&Touch2Share. One reason was the setup of the connection between phone and surface, which did not always work on the first attempt. Second, holding the connection between phone and surface was perceived as exhausting as users could not move the phone without risking disconnecting phone and surface. In addition, Shield\&Share allowed users to interact with only one hand. Select\&Place2Share and Select\&Touch2Share received the best results in terms of performance, effort, frustration, physical demand, and mental demand. 


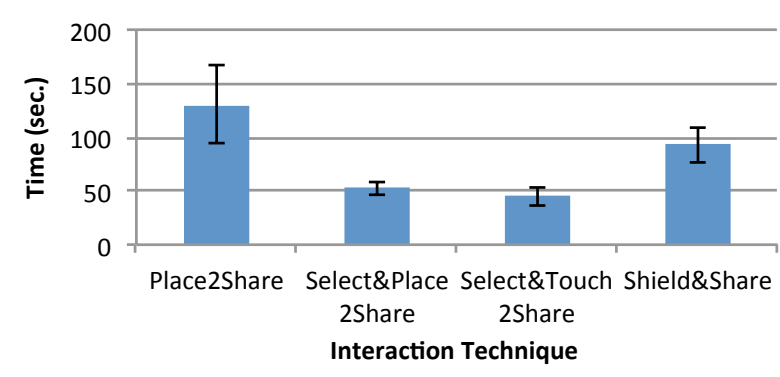

Fig. 16 Task completion times of the four interaction techniques.

For each interaction technique, participants rated how time consuming they felt the interaction technique was and how much the corresponding technique caused interruptions in the flow of interactions ( $5=$ very much). The results indicate a tendency that Select\&Place2Share was perceived as the fastest interaction technique $(M=1.98 ; S D=1.16)$. Select\&Touch2Share was rated with an average of $2.75(S D=0.95)$ and Shield\&Share with $3.0(S D=0.81)$. Place2Share was rated as the most time consuming technique $(M=3.25 ; S D=$ $1.70)$.

The feedback from the participants match the results from the measured task completion times. Figure 16 shows the mean task completion times of the different interaction techniques. A repeated measures ANOVA with a Greenhouse-Geisser correction determined that the difference in mean task completion time was statistically significant (at a significance level of $\alpha=0.05)$ for the four tested interaction techniques $(F(1.50,22.52)=16.33, p<0.001)$. Pairwise comparing through post-hoc tests using the Bonferroni correction reveals that the mean task completion time using the interaction technique Select\&Place2Share is significantly shorter than with Place2Share $p=0.005)$. Also, Select\&Touch2Share allows for significantly faster interaction times than Place2Share $(p=0.002)$. Yet, interaction with Shield\&Share was not significantly faster as with Place2Share $(p=0.217)$. The difference between the two fastest techniques, Select\&Place2Share and Select\&Touch2Share, is not significant $(p=0.09)$. However, Select\&Place2Share $(p=0.008)$ and Select\&Touch2Share $(p=0.001)$ are both significantly faster than Shield\&Share.

After completing the practical tasks, the participants ranked the four tested interaction techniques regarding which technique they considered as the fastest one in direct comparison to the others. They gave four points for the best and one point for the least preferred interaction technique. The best average score was reached by Select\&Place2Share (3.44 points), followed by Select\&Touch2Share with 3.25 points. Shield\&Share reached a score of 1.69 and Place2Share a score of 1.63 points.

Participants ranked on average Select\&Touch2Share (3.50 points) and Select\&Place2Share (3.44 points) as the best techniques for hiding private photos when sharing with other people. Shield\&Share reached a score of 2.06 points in this ranking and Place2Share only 1.00, which means that all participants ranked this technique to be the least suitable for protecting their privacy. Further, we asked the participants to rank the techniques regarding their suitability to be used for sharing single photos. The majority ranked Select\&Touch2Share as the best technique (in average 3.44 points) and Place2Share as the least suitable technique (1.06 points). Select\&Place2Share and Shield\&Share scored 2.75 and 2.13 points.

The ranking results of the interaction techniques' ability to support sharing of several photos in a sequence are more diverse. Select\&Place2Share (in average 3.44 points) and Select\&Touch2Share (2.75 points) were ranked as the best techniques. While Place2Share reached 2.13 points Shield\&Share received 1.69 points in this ranking.

Usability and Ease of Use. Participants gave diverse feedback regarding how well the interaction techniques supported them in sharing photos on the surface. For instance, several participants stated that they liked how easy it is to transmit photos to the surface when using Place2Share. One participant stated "I like that you don't need to configure anything before sharing images". Another pointed out that it is positive that "you can see all images on the surface right away". On the other hand, other feedback indicates issues of Place2Share: "It takes long until all photos are uploaded to the surface". In fact, it took around 5 seconds until all images were displayed on the surface. In practice, Place2Share would suffer from additional delays as all the images have to be transferred (e.g., via Bluetooth or $\mathrm{Wi}-\mathrm{Fi}$ ) between the devices once the phone is placed on the surface. Additionally, it was commented that "it is hard to find a specific photo amongst the others on the surface". One participant even pointed out that "after searching all the photos on the surface, my finger was burning". Also several participants criticized the fact that uploading all photos to the surface causes the screen to be cluttered.

Participants indicated that they liked the high-resolution preview on the phone screen when using Shield\&Share. Also the navigation through the photo albums using the controls on the surface were perceived as positive as well as the sharing and collecting of photos 


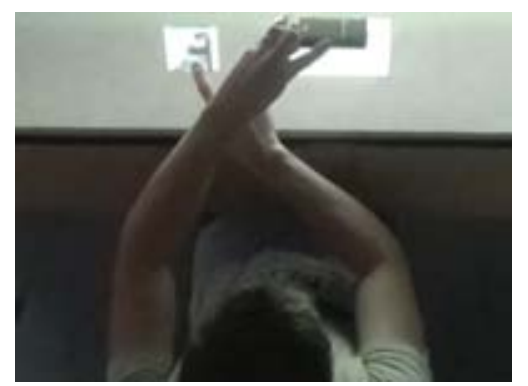

Fig. 17 Participant using Shield\&Share. The hand holding the phone is interfering with the interacting hand.

through dragging them out of (or into) the thumbnail bar onto the surface area. One participant highlighted that "this technique is great for sharing several photos spontaneously as I can make a selection and drag the photo on the surface". On the other hand, participants criticized that it was burdensome and tiring to hold the phone constantly in one hand. Some indicated that they did not like the low-resolution thumbnails on the surface so they often had to use the preview function on the phone screen. One major issue that came up is that the hand holding the phone can interfere with the interacting hand, see Figure 17.

Concerning Select\&Touch2Share, participants highlighted that they liked that the selection of the photos to share is done while the phone is in the hand of the user. They indicated that is was easy to share and to pick up photos from the surface. One participant stated "it is fast and the phone does not occlude on the surface". On the downside, one participant criticized that this technique requires touching the surface often with the phone which might damage the phone over time. Also, one participant criticized that "holding the phone in the hand all the time is positive but also a problem at the same time", indicating that only one hand is available for interacting with the photos on the surface.

Participants pointed out that it is positive that Select\&Place2Share allows the selection of photos to share before the phone is placed on the surface. They also appreciated having two hands available for interacting with the photos on the surface. One participant reported that "it is great that one can easily add photos from the surface to the phone", also applying to Place2Share which follows the same approach.

Privacy Support. Participants also gave rich feedback concerning the ability of each interaction technique to support the user protecting their privacy. With respect to Place2Share, participants gave exclusively negative feedback. For instance, one participant stated "photos that I did not intend to share were visible on the surface and others knew how many photos I have stored on my phone". Several participants indicated that they were missing a means for showing and sharing only selected photos.

Participants appreciated the ability of Shield\&Share to protect the user's privacy. For instance, one participant stated that "the low resolution thumbnails on the surface do not really reveal private information". They also highlighted that the preview on the phone screen allows for private access to photos. On the other hand, other participants criticized that the thumbnails can be seen by other users that are standing very close by. For instance, one participant criticized that "people standing around can see thumbnails of my private data easily". On the other hand, another participant stated that "the thumbnails in the menu bar are very blurry. I often had to use the preview on the phone to check what photo it actually was".

Participants mentioned regarding Select\&Touch2Share that it is great that the selection of photos is done in private while the phone is held in the hand. One participant stated "it was easy and fast to use. Others cannot see how many photos I have stored on my phone and I could decide whether I share one or more photos at a time" and "this technique is ideal for selecting specific photos from a set of personal photos". Concerning the effectiveness of Select\&Touch2Share to support the user's privacy one participant pointed out that "private photos are not revealed to others at all. I can check my selection before I transmit the photos to the surface". The feedback concerning Select\&Place2Share contained similar aspects. Users liked the selection of photos beforehand and the good privacy protection support. However, they pointed out that it is a problem when placing the phone on the surface when a photo album containing private photos is visible on the phone: "you have to be careful that the public folder is visible on the phone when placing it on the surface. Otherwise private photos can be visible to others". This aspect was not considered in the implementation but could be fixed easily. For example, the screen could be turned off automatically as the phone is placed on the surface. With respect to how effective Select\&Place2Share supports the user's privacy, users stated that they liked the "silent" selection of photos that is made in private. However, one user criticized that using Select\&Place2Share would not support to share several photos after another: "using this technique it makes more sense to select all photos that you want to share otherwise you have to pick up the phone each time you want to share additional photos".

Most of the participants indicated with their feedback that they were aware of privacy issues in the con- 
text of photo sharing and that privacy is important to them. For instance, one participant stated "if I could not hide my private photos, I would not share any". In addition, participants pointed out that sharing selected photos is more usable: "it is very annoying to search on the surface for certain photos!"

\section{Discussion and Conclusion}

Interactive surfaces are great devices for collaborative work as multiple users can view and interact with contents simultaneously. For personalization, personal devices such as mobile phones can be integrated enabling seamless access to personal data. Users can then easily share and exchange photos, contacts, and other kinds of data or files. However, users often store large amounts of data on their personal mobile devices. Considerable parts of the data can be regarded private and even highly sensitive, for instance, specific pictures, text messages, or notes. Therefore, interaction techniques for sharing and exchanging data from the personal mobile phone need to support the users and protect their privacy. That is, they need to be privacy respecting.

An increasing number of social networks enable users to share their photos with their friends and communities. For instance, Facebook or Twitter support quick sharing of photos through different mobile application. Vice versa, users have access to a constantly growing amount of photos that were uploaded by their contacts. When accessing photos from social network sources for sharing them in a face-to-face context from the mobile phone on an interactive surface, users require even more effective means for selecting which photos are displayed on the shared interactive surface. The main difference to accessing photos stored on the personal device is that users cannot control which data is shared and appears in the stream of photos. As a result, the amount of shared photos that are potentially irrelevant in the current sharing situation increases. Also, photos that are not appropriate in the current situation could be uploaded to the social network media streams. Therefore, when accessing photos from social networks and sharing them with present persons, users benefit from means provided by the presented interaction techniques that allow users to select which data they want to disclose. The necessity of filtering data in the context of social networks is reflected, for instance, by the concept of Circles in Google+.

In this work, we investigated three interaction techniques (Select\&Place2Share, Select\&Touch2Share, and Shield\&Share) which allow users to select and control which data they share with others on an interactive surface and thus support users to protect their privacy. In addition, we considered the interaction technique Place2Share that has been reported and demonstrated previously, which enables straightforward data sharing but does not provide any kind of privacy support.

\begin{tabular}{|c|c|c|c|}
\hline & $\begin{array}{l}\text { Item Selection } \\
\text { Time }\end{array}$ & $\begin{array}{l}\text { Location of } \\
\text { phone }\end{array}$ & $\begin{array}{l}\text { Sequential } \\
\text { Sharing }\end{array}$ \\
\hline $\mathrm{P} 2 \mathrm{~S}$ & - & On surface & -- \\
\hline SP2S & Before & On surface & - \\
\hline ST2S & Before & In hand & + \\
\hline $\mathrm{S} \& \mathrm{~S}$ & During & $\begin{array}{l}\text { In hand \& on } \\
\text { surface }\end{array}$ & ++ \\
\hline
\end{tabular}

Table 1 Comparison of sharing interaction techniques Select\&Place2Share (P2S), Select\&Place2Share (SP2S), Select\&Touch2Share (ST2S), and Shield\&Share (S\&S)

These interaction techniques differ in particular regarding the time of data selection, the phone location during interaction, and to what extend they support users in sharing multiple data items sequentially one after another (see Table 1). Place2Share does not support selecting items for sharing and due to the phone being placed on the surface, interaction with the phone is difficult to perform. Accordingly, sequential sharing of different data items is only possible in terms of pointing out different items. Yet, all items are transferred at the same time. However, this technique can be suited in application context where the data items that are to be shared is determined through additional logic. For instance, in a card game context (see [3]), the mobile phone could be used for displaying the user's cards. For showing the cards to other players, the phone could simply placed on the shared surface and corresponding cards are displayed around the phone. However, this technique appears to be not suitable in application contexts in which no logic can determine the selection of items, which will be disclosed. Hence, Select\&Place2Share is better suited in application contexts when large numbers of potential items are available such as the case of photo collections. Yet, this technique requires, as the previous, to place the phone on the surface, which makes it difficult to interact with the phone. For instance, when selecting additional items for sharing them on the surface. Therefore, the technique Select\&Touch2Share, which allows the users to keep the mobile phone in her hand throughout the interaction is more suited for application scenarios, in which users share multiple items sequentially as it might be the case when for instance, giving report on a journey. In contract to the previous techniques, Shield\&Share allows making the selection continuously as the user holds on the phone that is touching the surface. On the down- 
side, one hand of the user is constantly blocked for interaction which might result in fatigue. Hence, mobile phones that are equipped with a stand (e.g., the HTC HD7) which allows the phone to remain in an upright position without the user's help, are potentially more suited. This would enable not only sharing items such as photos but also applications such as giving presentations to customers sitting around an interactive surface.

We evaluated these four interaction techniques in a comparative user study. In particular we focused on how users perceived the tested interaction techniques in terms of interaction speed, usability, and in particular how well each of the techniques supports users to disclose only specific selected photos.

The main findings from the evaluation are that (1) users prefer interaction techniques that enable making a selection of what data are to be shared. (2) Users prefer selecting the data before an interaction with the interactive surface starts. (3) The ability to easily share several photos in a sequence and not all at the same time is important to users. The interaction techniques Select\&Place2Share and Select\&Touch2Share allowed participants in our study with 16 participants to perform significantly faster compared with Shield\&Share and Place2Share. Select\&Touch2Share requires users to often touch the surface with their mobile phone, which was reported to be something they would not like to do too often with their own mobile phones. Therefore, Select\&Place2Share can be seen as a suitable alternative as it supports protecting privacy at a similar level. Shield\&Share turned out to be hard to use and tiring because users had to hold the phone constantly with one hand while performing the interaction with the other. We can conclude that Shield\&Share is not ideal in the setting as applied in the user study, yet it could have a positive impact in other areas of application such as gaming.

In this work, the implementation and evaluation focused on sharing of photos as one example. As different kinds of data place different demands in terms of privacy and access rights, we are planning to consider how other kinds of data (e.g., documents, calendars, or contacts) affect the way how users want to share them with others using surfaces. For instance, when arranging a meeting using an interactive surface, it is likely that users do not want to display all their calendar entries on the surface. Based on the given situation and context, users should be able to control what information is displayed and at what level of detail. It is open to question if users would prefer to disclose available time slots visualized on the surface, or if only selected appointments should transferred to the surface.
Acknowledgements This work was conducted in the Emmy Noether research group Mobile Interaction with Pervasive User Interfaces funded by the German Research Foundation (DFG).

\section{References}

1. Ballagas, R., Borchers, J., Rohs, M., Sheridan, J.: The smart phone: a ubiquitous input device. Pervasive Computing, IEEE 5(1), 70 - 77 (2006). DOI 10.1109/MPRV. 2006.18

2. Chehimi, F., Rukzio, E.: Throw your photos: an intuitive approach for sharing between mobile phones and interactive tables. In: Ubicomp '10 (adjunct proceedings), pp. 443-444. ACM (2010). DOI http://doi.acm.org/10.1145/ 1864431.1864479

3. Döring, T., Shirazi, A.S., Schmidt, A.: Exploring gesturebased interaction techniques in multi-display environments with mobile phones and a multi-touch table. In: Proceedings of the International Conference on Advanced Visual Interfaces, AVI '10, pp. 419-419. ACM, New York, NY, USA (2010)

4. Greenberg, S., Boyle, M., Laberge, J.: PDAs and shared public displays: Making personal information public, and public information personal. PUC 3, 54-64 (1999)

5. Han, J.Y.: Low-cost multi-touch sensing through frustrated total internal reflection. In: UIST '05, pp. 115-118. ACM (2005)

6. Hart, S., Stavenland, L., Hancock, P., Meshkati, N.: Development of NASA-TLX (Task Load Index): Results of empirical and theoretical research. In: Human Mental Workload, pp. 139-183. Elsevier (1988). URL http://ntrs.nasa.gov/archive/nasa/casi.ntrs. nasa.gov/20000004342_1999205624.pdf

7. Hinckley, K., Ramos, G., Guimbretiere, F., Baudisch, P., Smith, M.: Stitching: pen gestures that span multiple displays. In: AVI '04, pp. 23-31. ACM (2004)

8. Izadi, S., Brignull, H., Rodden, T., Rogers, Y., Underwood, M.: Dynamo: a public interactive surface supporting the cooperative sharing and exchange of media. In: Proceedings of the 16th annual ACM symposium on User interface software and technology, UIST '03, pp. 159-168. ACM, New York, NY, USA (2003)

9. Kim, D., Dunphy, P., Briggs, P., Hook, J., Nicholson, J., Nicholson, J., Olivier, P.: Multi-touch authentication on tabletops. In: CHI '10, pp. 1093-1102. ACM (2010)

10. Kyocera Communications, Inc.: Kyocera echo dual-touchscreen android smartphone. http://www. echobykyocera.com/ (2011). (Last Access: 2012/10/02)

11. Microsoft: See surface applications in action. http://www.microsoft.com/surface/en/us/ applicationpages10.aspx (2011). (Last Access: 2012/10/02)

12. Microsoft Surface media sharing scenario.: http://www . youtube. com/watch?v=zVdhrnWqOvY (2011). (Last Access: 2012/10/02)

13. Mobile Connect Sample Application: MS TechNet. http://technet.microsoft. com/en-us/library/ ee692087 (Surface.10).aspx (2011). (Last Access: 2012/10/02)

14. Myers, B.A.: Using handhelds and PCs together. Commun. ACM 44, 34-41 (2001)

15. Rekimoto, J.: Pick-and-drop: a direct manipulation technique for multiple computer environments. In: UIST ' 97 , pp. 31-39. ACM (1997) 
16. Rekimoto, J.: A multiple device approach for supporting whiteboard-based interactions. In: CHI '98, pp. 344-351. ACM Press/Addison-Wesley Publishing Co. (1998)

17. Schmidt, D., Chehimi, F., Rukzio, E., Gellersen, H.: PhoneTouch: a technique for direct phone interaction on surfaces. In: UIST '10, pp. 13-16. ACM (2010)

18. Schmidt, D., Seifert, J., Rukzio, E., Gellersen, H.: A cross-device interaction style for mobiles and surfaces. In: Proceedings of the Designing Interactive Systems Conference, DIS '12, pp. 318-327. ACM, New York, NY, USA (2012)

19. Seewoonauth, K., Rukzio, E., Hardy, R., Holleis, P.: Two nfc interaction techniques for quickly exchanging pictures between a mobile phone and a computer. In: MobileHCI '09, pp. 39:1-39:4. ACM (2009). DOI http://doi.acm. org/10.1145/1613858.1613909

20. Seifert, J., Luca, A., Conradi, B., Hussmann, H.: Treasurephone: Context-sensitive user data protection on mobile phones. In: P. Floren, A. Krger, M. Spasojevic (eds.) Pervasive Computing, Lecture Notes in Computer Science, vol. 6030, pp. 130-137. Springer Berlin Heidelberg (2010). DOI 10.1007/978-3-642-12654-3_8. URL http://dx.doi.org/10.1007/978-3-642-12654-3_8

21. Shen, C., Everitt, K., Ryall, K., Dey, A., Schmidt, A. McCarthy, J.: UbiTable: impromptu Face-to-Face collaboration on horizontal interactive surfaces. In: UbiComp 2003, pp. 281-288. Springer (2003)

22. Shoemaker, G.B.D., Inkpen, K.M.: Single display privacyware: augmenting public displays with private information. In: CHI '01, pp. 522-529. ACM (2001)

23. Wilson, A.D., Sarin, R.: Bluetable: connecting wireless mobile devices on interactive surfaces using vision-based handshaking. In: GI '07, pp. 119-125. ACM (2007)

24. Wu, M., Balakrishnan, R.: Multi-finger and whole hand gestural interaction techniques for multi-user tabletop displays. In: UIST '03, pp. 193-202. ACM (2003). DOI http://doi.acm.org/10.1145/964696.964718 